\title{
SIMULAÇÃO DO SOFTWARE ARENA PARA O ABASTECIMENTO DE REFRIGERANTES EM UMA REDE DE SUPERMERCADOS NO MUNICÍPIO DE BEBEDOURO (SP)
}

\author{
SIMULATION OF ARENA SOFTWARE TO SUPPLY COOLANTS IN A \\ SUPERMARKET NETWORK IN THE CITY OF BEBEDOURO (SP)
}

\author{
Paulo Tiago Dionizio - paulo-tiago85@ hotmail.com \\ Rhadler Herculani - rhadler.herculani@ fatecbb.edu.br \\ Faculdade de Tecnologia de Bebedouro (FATEC) -SP -Brasil
}

DOI: 10.31510/infa.v16i2.634

\section{RESUMO}

O objetivo do presente trabalho se constitui em utilizar o software ARENA para simulação e otimização de tempo total gasto com a atividade de abastecimento de refrigerantes em uma rede de supermercados no município de Bebedouro (SP). Com a finalidade de otimizar a atividade logística e diminuir o tempo de atendimento aos clientes, fez-se necessário a cronometragem de todo o processo laboral de um funcionário colaborador para esse estudo, desde a saída de sua residência para o início do expediente, até o término do último abastecimento previsto para o mesmo dia, no total de 19 dias cronometrados, com o propósito de conhecer bem o funcionamento de toda cadeia de transporte, a fim de que se consiga realizar um bom planejamento que atenda às necessidades da empresa, ao mesmo tempo em que seja capaz de reduzir os custos de execução da atividade de abastecimento e obtenha êxito em atender as expectativas dos seus clientes. A distribuição física dos produtos deve ser realizada sempre buscando abastecer, no tempo certo e com a qualidade desejada (ou acima do esperado) clientes finais do varejo, no caso uma rede de supermercados para o município de Bebedouro (SP). Conseguir manter um padrão de excelência e de qualidade entre todas as etapas da cadeia de abastecimento será um diferencial almejado e que resultará na fidelidade para com os serviços prestados.

Palavras-chave: Abastecimento. Distribuição. Otimização. Transporte.

\begin{abstract}
The objective of this work is to use the software ARENA to simulate and optimize the total time spent with the supply activity of soft drinks in a supermarket chain in the municipality of Bebedouro (SP). In order to optimize logistic activity and shorten customer service time, it was necessary to timing the entire labor process of a collaborating employee for this study, from the time of leaving his residence to the beginning of his Work, until the end of the last
\end{abstract}


supply scheduled for the same day, in the total of 19 timed days, in order to know well the functioning of the entire transportation chain, in order to achieve a good planning that meets the needs of the company, at the same time as it is able to reduce the costs of executing the supply activity and is successful in meeting the expectations of its customers. The physical distribution of the products must always be carried out in order to supply, at the right time and with the desired quality (or higher than expected) the final customers of the retail, in this case a supermarket chain of the municipality of Bebedouro (SP). Achieving a standard of excellence and quality among all stages of the supply chain will be a desired differential and will result in loyalty to the services provided.

Keywords: Supply.Distribution. Optimization. Transport.

\section{INTRODUÇÃO}

O segmento de bebidas se constitui em um mercado muito dinâmico no Brasil, justificado por um clima tropical e com calor na maior parte do ano, contribuindo assim para a demanda por bebidas geladas. Ao se analisar o segmento de refrigerantes no Brasil, nota-se que ele se encontra existente em praticamente todos os municípios do país. Nesse mercado cada vez mais competitivo, muitas empresas optam por oferecer um serviço de transporte que proporcione, como um diferencial competitivo, uma entrega com maior rapidez, segurança e agilidade, assegurando aptidão e pontualidade em suas atividades de abastecimento de refrigerantes em estabelecimentos comerciais (AMORIN, 2014).

O objetivo do presente trabalho se constitui em utilizar o software ARENA para simulação e otimização de tempo total gasto com a atividade de abastecimento de refrigerantes em uma rede de supermercados no município de Bebedouro (SP), justificado pelo fato de um dos autores desse artigo trabalhar na empresa analisada e, assim, facilitar a coleta de dados para o estudo em questão.

A escolha pelo segmento de refrigerantes se justifica pelo fato de as empresas que atuam nesse ramo possuírem algumas características particulares, como uma grande quantidade de produtos ofertados, elevada concorrência, preços baixos para esse tipo de produto oferecido e a questão da importância da marca para o cliente (DANTAS, 2000). Dessa forma, a descrição das etapas relacionadas a roteirização para o abastecimento dessa rede de supermercados permite que a empresa analisada possa saber quais serão os seus pontos a melhorar e, junto com isso, busque otimizar seu desempenho.

\section{FUNDAMENTAÇÃO TEÓRICA}

\subsection{Cadeia de abastecimentos e o setor de refrigerantes}

Segundo Araújo (2008), a cadeia de abastecimento diz respeito ao agrupamento dos procedimentos requeridos para a aquisição de materiais, adicionando-lhe valores segundo a 
concepção dos consumidores e disponibilizando-os no lugar e na data desejada pelos mesmos. A cadeia possui modelos que divergem segundo critérios do produto, do negócio e das estratégias usadas pelas empresas para que consigam realizar a entrega de um determinado bem para seus consumidores (BALLOU, 2006).

Otimizar a eficiência de uma cadeia de abastecimento corresponde a otimizar a eficiência da estrutura interna de uma empresa que a utiliza, assim como de suas ligações produtivas (relações entre duas ou mais empresas subseqüentes em um mesmo processo produtivo). Para isso, a construção dessa estrutura necessita de uma compreensão profunda dos procedimentos e de suas variáveis relacionadas, como por exemplo o conhecimento dos padrões de comportamento do mercado (com suas demandas específicas), os tipos de transporte usados, os canais de distribuição geográfica, a constituição dos custos associados a execução dessa atividade e outros itens de igual importância (ARAÚJO, 2008).

O segmento de refrigerantes é estabelecido tanto pelas circunstâncias de demanda quanto de oferta. A demanda é influenciada por aspectos demográficos, econômicos, socioculturais, ambientais e pelas circunstâncias de oferta. Os principais aspectos da demanda são hábitos de vida, produção de eventos, clima, renda per capita, gênero, idade e acessibilidade aos pontos de venda de bebidas (DONATO, 2013). Já sobre a oferta, as principais empresas desse segmento visam conseguir diversificação e diferenciação de produtos, como por exemplo o lançamento de refrigerantes com níveis de calorias, cafeína e açúcar baixos ou mesmo a apresentação de novos sabores para esses produtos (o que ajuda, com atitudes como essa, a fortalecer a marca perante os consumidores ao atender a uma maior variedade de gostos individuais).

A logística para esse ramo é dividida em três partes: dos fornecedores de matériasprimas, embalagens e insumos para as engarrafadoras; destes para centros de distribuição e revendedores (que podem fazer parte da própria indústria ou serem terceirizados); e destes para os locais finais de vendas (sejam varejistas ou atacadistas), como por exemplo supermercados, lojas de conveniência, mercearias ou hipermercados (DONATO, 2010). Para Mentzer et al (2001), a concorrência entre empresas hoje se define baseada em qualidade e tempo, ou seja, adquirir um produto sem defeitos, entregando-o ao consumidor da forma mais eficiente e rápida possível, não é mais visto como um diferencial competitivo, e sim como uma exigência do próprio mercado aonde a empresa atua. Dessa maneira, é fundamental que as empresas consigam responder as vontades do mercado no instante certo e na qualidade desejada.

Para Bertaglia (2003), muitos modelos de cadeia de abastecimento são complexos e isso torna essencial a identificação da quantidade de fornecedores e clientes na cadeia em questão. É importante localizar cada um deles para a execução das operações de abastecimento, assim como verificar a importância de cada um nos critérios de quantidade de compras e vendas. Uma boa gestão para a cadeia de abastecimento apresenta como objetivo final a otimização do processo produtivo para qualquer empresa que busque melhorá-la, ou de acordo com Van der Vorst et al (2007), mostrar uma relação entre demanda e oferta cujo propósito será o de adicionar valor à mercadoria ao menor custo viável de produção. 


\subsection{Simulação e o seu desenvolvimento histórico}

A utilização moderna da simulação remonta sua origem para o Método de Monte Carlo (1940). Para Saraiva Júnior et al. (2011), esse método consiste em utilizar números aleatórios e probabilísticos para a análise e resolução de problemas. O uso desse método para simulação pode ser empregado em problemas de tomada de decisão em que estejam inclusos a incerteza e o risco, ou seja, circunstâncias nas quais o desempenho das variáveis relacionadas com o problema não é de natureza determinística (FRANCISCHETTI, 2015).

Com o surgimento dos primeiros computadores, em meados dos anos 50, a idéia do Método de Monte Carlo foi ampliada para a solução de problemas probabilísticos de natureza mais ampla, como por exemplo a existência de filas de espera. Dessa forma, nascia a simulação de Monte Carlo (SALIBY, 1989). A partir de então, a simulação passou a ser uma área de estudo cada vez mais usada nas mais diversas áreas de conhecimento. Dois motivos influenciaram para isso: o aumento da complexidade dos problemas a serem solucionados e a uma maior oferta de recursos computacionais.

Com o advento do desenvolvimento tecnológico observado, opta-se então pelo uso do recurso computacional da simulação como ferramenta para o estudo das respostas de um sistema, sem que seja necessário a construção deste ou mesmo alterá-lo (FERREIRA, 2006). Com isso, se torna frequente a utilização de softwares computacionais como Arena, Flexsim, Promodel, Símio, dentre outros, para a elaboração de um cenário virtual propício para o estudo pretendido.

Para fins do presente artigo, um software bastante usado e muito eficiente, e que será abordado nesse trabalho, é o ARENA. Esse software possui todos os recursos fundamentais para animações, modelagem de processos e análise de resultados. A elaboração do processo de simulação é visual, o que facilita bastante a modelagem. Uma das vantagens em utilizá-lo, segundo Paragon (2005) é que, além da elaboração do modelo e o alcance dos dados pertinentes ao processo de simulação, é possível também elaborar animações (um recurso fundamental que simplifica a divulgação do modelo concebido e também a visualização do processo).

\subsection{O software arena e a teoria de simulação}

Toda simulação exige a concepção de um modelo com o qual serão realizados os experimentos. Um modelo matemático compreendido por meio da simulação é denominado de modelo de simulação (SALIBY, 1989).

Para Barton (1970), um modelo de simulação possui as seguintes características:

1. Objetivo de reproduzir a totalidade ou parte de um sistema;

2. Viabilidade de ser realizado ou alterado; 


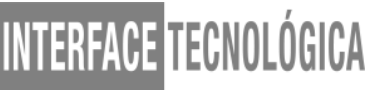

3. Possuir o tempo ou um contador de repetições como uma de suas variáveis;

4. Apresentar um argumento que auxilie na compreensão de um sistema como um todo.

O software ARENA, que será usado no presente artigo, é composto pela união dos recursos de uma linguagem de simulação à facilidade de utilização de um simulador, em um cenário gráfico incluso. A concepção de simulações no ARENA é um trabalho geralmente fácil, pois basta a formulação de um modelo lógico-matemático para a representação do sistema que está sendo compreendido. Para esse modelo, devem existir valores para a denominação de distâncias, recursos e os tempos que estão disponíveis para a simulação (PAULA; HAIDUKE, 2015).

Há duas categorias de módulos no ARENA, que são o módulo de fluxogramas e os módulos de dados.

De acordo com Prado (2014) o módulo de fluxogramas é utilizado para a construção dos modelos lógicos e de animação

A estruturação do módulo Basic Process se inicia pela Área de Templates (localizada no lado esquerdo da tela). Para ativar algum template, basta clicar com o mouse no modelo desejado, e arrastá-lo até a área de trabalho.

A programação lógica é concebida na área de trabalho através dos módulos e conectores, formando, desta forma, um fluxograma do sistema. Cada bloco do fluxograma retrata uma ocorrência (por exemplo: início, operação e término) e este equivale a um módulo de um dado (template) para o ARENA 15.0.

Já o módulo de dados consiste na reunião de dados referentes ao modelo, mas que não são inseridos no processo operacional (PRADO, 2014). Dessa forma, nesse módulo se encontram as seguintes informações principais de suporte para análise:

- ENTITIES - agrupa as definições e fatores relacionadas a todas as formas de entidades utilizadas no modelo. A inserção de dados é executada por meio de uma caixa de diálogo ou área de planilha;

- $Q U E U E$ - permite a prioridade para uma tarefa por meio do valor conferido a mesma;

- RESOURCE - determina os recursos no sistema de simulação, com a inclusão de dados sobre custos e disponibilidade de recursos.

E por fim, após concluído o fluxograma, porém antes de pedir a execução do modelo, deve-se oferecer as opções de controle da execução. Para tal propósito, deve-se clicar em Rodar e ir no menu Configurar, completando adequadamente os seguintes campos:

- Delimitar o número de replicações desejadas para o modelo em questão na opção "Número de Replicações", que se encontra disponível na aba "Parâmetros de Replicação". 


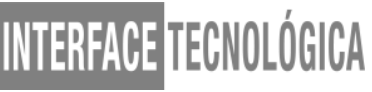

- Poder escolher que haja a execução do modelo por um determinado período de tempo sem coleta de dados estatísticos na opção "Período de Aquecimento".

- Determinar que o modelo seja interrompido depois de transcorridos um determinado período de tempo na opção "Duração da Replicação".

- Ponderar quantas horas por dia serão levadas em consideração no modelo na opção "Horas por Dia".

- Delimitar qual será a unidade de tempo base em que será baseada a apresentação dos relatórios de simulação na opção "Unidade de Tempo Base".

Como complemento, deve-se ressaltar a importância do Input Analyzer (IA) como ferramenta dentro do software ARENA que viabiliza o estudo dos dados reais do andamento de um processo e opta pela melhor distribuição estatística que se enquadre a eles (PRADO, 2010). O recurso pode ser acessado por meio da opção Ferramentas, e selecionando em seguida a opção Input Analyzer.

Para conceber um arquivo com dados a serem examinados pelo IA, deve-se coletar os tempos aleatoriamente no processo escolhido para ser gera a simulação.

Após isso, deve-se copiar tempos coletados em um programa de edição de textos, como o bloco de notas por exemplo e salvá-los em uma pasta.

Ao voltar para o IA, acesse a opção Novo, em seguida selecione Use Arquivo de Dados Existente e procure pelo arquivo salvo em formato do programa de edição de textos (o formato será o ".txt"). Em seguida, acesse a opção Fit e selecione Fit All (esse item irá mostrar a distribuição que melhor se adapta àquela concebida pelos dados). Assim, deverá ser encontrado a curva de distribuição desejada para a atividade de simulação em questão.

E, por fim, na parte abaixo do painel, irá aparecer as informações sobre essa curva de distribuição encontrada (seu nome e seus parâmetros). Serão esses dados então que irão ser utilizados como dados de entrada para o modelo de simulação em questão no software ARENA.

\section{MATERIAIS E MÉTODOS}

Com o objetivo de mensurar o tempo gasto na atividade de abastecimento em uma rede de supermercados para o município de Bebedouro (SP), no intuito de averiguar possíveis problemas logísticos com relação a possibilidade de melhoria de tempo total gasto com a execução dessa atividade, o grupo utilizou de pesquisa exploratória, de caráter qualitativo, tendo como base o acompanhamento in loco da operação de abastecimento desses estabelecimentos por uma distribuidora de refrigerantes em dezenove dias laborais e uma pesquisa bibliográfica sobre esse tipo de atividade. Com os dados obtidos, propôs-se a utilizar 
o software ARENA para compreensão de resultados e sugestões de melhorias que propiciem a otimização da atividade de abastecimento em questão.

De início, as somatórias dos tempos coletados para os respectivos dias se encontram na Tabela 1 a seguir:

Tabela 1 - Somatória dos tempos cronometrados para a atividade de abastecimento em supermercados no município de Bebedouro (SP)

Tempos de Cronometragem entre os dias 30/08 ao 20/09 (Supermercados de Bebedouro) - Em Minutos

\begin{tabular}{|c|c|c|c|c|c|c|c|c|}
\hline $\begin{array}{c}\text { Laranjão S. } \\
\text { (Rodoviária } \\
\text { ) }\end{array}$ & $\begin{array}{c}\text { Extra } \\
\text { Supermercad } \\
\text { o }\end{array}$ & $\begin{array}{c}\text { Laranjão } \\
\text { S. } \\
\text { (Centro) }\end{array}$ & $\begin{array}{c}\text { Savegnag } \\
\text { o S. (Loja } \\
\text { 21) }\end{array}$ & Freitas $\mathrm{S}$. & $\begin{array}{c}\text { Savegnag } \\
\text { o S. (Loja } \\
\text { 18) }\end{array}$ & $\begin{array}{c}\text { Iquegami S. } \\
\text { (Centenário } \\
\text { ) }\end{array}$ & $\begin{array}{c}\text { Sesé } \\
\text { Supermercad } \\
\text { o }\end{array}$ & $\begin{array}{l}\text { Iquegami } \\
\text { S. (Vila } \\
\text { Paulista) }\end{array}$ \\
\hline 45.62 & 52.72 & 32.42 & 37.47 & 14.73 & 29.13 & 29.87 & 39.85 & 125.00 \\
\hline 22.90 & 27.65 & 27.65 & 27.20 & 15.03 & 25.18 & 27.65 & 25.33 & 54.02 \\
\hline 65.13 & 58.72 & 58.72 & 25.38 & & 20.3 & 65.22 & 38.83 & 64.03 \\
\hline 52.62 & 22.58 & 22.58 & 21.80 & & 22.03 & 25.35 & 27.33 & 51.02 \\
\hline 17.85 & 52.47 & 52.47 & 30.03 & & 20.68 & 54.05 & 24.27 & 88.13 \\
\hline 67.13 & 27.15 & 27.15 & 32.50 & & 32.42 & 67.22 & 70.38 & 270.13 \\
\hline 46.80 & & & 30.20 & & 32.18 & 28.53 & & 127.03 \\
\hline 22.98 & & & 47.43 & & 42.35 & 59.75 & & 59.20 \\
\hline \multirow[t]{9}{*}{64.02} & & & 16.77 & & 17.13 & 64.38 & & 46.03 \\
\hline & & & 32.03 & & 22.68 & 45.28 & & 81.13 \\
\hline & & & 35.35 & & 35.27 & & & 273.35 \\
\hline & & & 24.05 & & 26.05 & & & 50.32 \\
\hline & & & 56.28 & & 51.62 & & & 64.37 \\
\hline & & & 21.03 & & 22.23 & & & 51.17 \\
\hline & & & 29.72 & & 19.97 & & & 89.20 \\
\hline & & & 29.15 & & 15.35 & & & 267.42 \\
\hline & & & & & & & & 142.53 \\
\hline
\end{tabular}

Fonte - Autoria Própria

Utilizando-se a ferramenta do Input Analyzer, disponível no software ARENA, obteve-se as seguintes expressões para cada processo, conforme a tabela 2:

Tabela 2 - Expressões de entrada dos processos

Savegnago Loja 21 Bebedouro

Supermercado Freitas Bebedouro
$16+\operatorname{GAMM}(7.41,2.03)$

$14.7+0.36 * \operatorname{BETA}(0.596$ 
$0.618)$

\begin{tabular}{ll}
\hline Savegnago Loja 18 Bebedouro & $15+\operatorname{EXPO}(12.2)$ \\
\hline Iquegami S. Centenário Bebedouro & $25+43 * \operatorname{BETA}(0.546,0.59)$ \\
\hline Sesé Supermercado Bebedouro & $24+\operatorname{EXPO}(13.7)$ \\
\hline Iquegami S. Vila Paulista Bebedouro & $46+228 * \operatorname{BETA}(0.336,0.562)$ \\
\hline Laranjão S Loja 1 Rodoviaria & $17+51 * \operatorname{BETA}(0.64,0.56)$ \\
\hline Extra Supermercado & $22+37 * \operatorname{BETA}(0.487,0.474)$ \\
\hline Laranjão S. Loja 2 Centro & $22+\operatorname{LOGN}(19.7,46.7)$ \\
\hline
\end{tabular}

Fonte - Autoria Própria

E, por fim, o fluxograma para a atividade de abastecimento realizada para cada supermercado se estruturou de acordo com a figura 1:

Figura 1 - Fluxograma para a atividade de abastecimento nos supermercados do município de Bebedouro (SP)

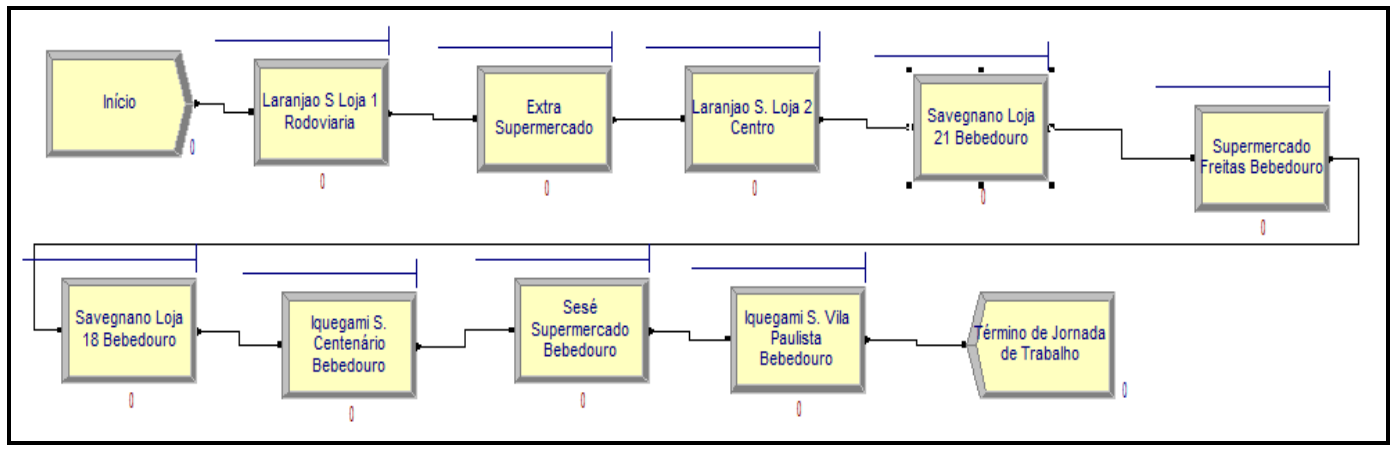

Fonte - Autoria Própria 
Figura 2 - Relatório do Tempo das Filas (Queues)

\begin{tabular}{|c|c|c|c|c|c|}
\hline Replication 1 & 0,00 & Stop Time & 480,00 & Time Units: & Minutes \\
\hline \multicolumn{6}{|l|}{ Queue Detail Summary } \\
\hline \multicolumn{6}{|l|}{ Time } \\
\hline & & Waiting Time & & & \\
\hline Extra Supermercado.Queue & & 3.15 & & & \\
\hline Iquegami S.Centenário Bebedouro.Queue & & 5.15 & & & \\
\hline Iquegami S.Vila Paulista Bebedouro.Queue & & 24.72 & & & \\
\hline Laranjao S Loja 1 Rodoviaria Queue & & 59.00 & & & \\
\hline Laranjao S.Loja 2 Centro.Queue & & 10.86 & & & \\
\hline Savegnano Loja 18 Bebedouro.Queue & & 10.48 & & & \\
\hline Savegnano Loja 21 Bebedouro.Queue & & 9.87 & & & \\
\hline Sesé Supermercado Bebedouro.Queue & & 9.49 & & & \\
\hline Supermercado Freitas Bebedouro.Queue & & 0.00 & & & \\
\hline
\end{tabular}

Fonte - Autoria Própria

\section{RESULTADOS E DISCUSSÃO}

Após a simulação realizada no software ARENA, verificou-se a existência de gargalos nos supermercados Laranjão (Rodoviária) e Iquegami (Vila Paulista), conforme os tempos de filas na figura 2, o qual relatou filas de 59 minutos e de quase 25 minutos, respectivamente:

A observação constatada de um maior tempo destinado ao abastecimento nos supermercados Laranjão (Rodoviária) e Iquegami (Vila Paulista) é justificada pelos respectivos estabelecimentos comerciais apresentarem um maior quantidade de produtos, além dos refrigerantes, para serem abastecidos nesses lugares. No supermercado Laranjão (Rodoviária), os produtos se encontram inicialmente no subsolo, aonde necessitam utilizar um elevador para os içar até a loja, o que demanda um tempo extra associado à demora ao abastecimento deste estabelecimento comercial. E, ao verificar o supermercado Iquegami (Vila Paulista), notou-se que a grande distância a ser percorrida do depósito para a loja em si faz com que se agregue um tempo extra para a atividade de abastecimento deste estabelecimento comercial, com a possibilidade deste tempo ainda ser elevado devido à grande quantidade de produtos a serem abastecidos nesse local, além dos refrigerantes, o que ocasiona a demora averiguada na execução da atividade em questão.

Como sugestão de melhoria, ambos os estabelecimentos comerciais necessitam de investimentos em sua estrutura, para que assim, agilizem o abastecimentos, reduzindo a espera dos funcionários da empresa distribuidora e dos respectivos estabelecimentos, tornado esse processo mais ágil e consistente com os padrões de logística mundial (ARAÚJO, 2008). 


\section{CONCLUSÃO}

Com o uso do software ARENA para a simulação da atividade de abastecimento nos supermercados do município de Bebedouro (SP), identificou-se a existência de gargalos operacionais para a execução desta respectiva atividade nos supermercados Laranjão (Rodoviária) e Iquegami (Vila Paulista).

A solução para os problemas identificados passaria por se utilizar maiores recursos tecnológicos para a diminuição do tempo gasto na execução da atividade (como, por exemplo, o uso de tablets ou aplicativos de celulares para a minimização de tempo gasto com burocracia), o que levaria, consequentemente, a uma redução do tempo de espera no reabastecimento desses supermercados. E, além disso, a criação de roteiros flexíveis para o abastecimento, mediante as necessidades mais urgentes de demanda, propiciaria uma economia de custos operacionais junto com minimização dos tempos de execução da atividade em questão.

Os resultados desta análise expõem a relevância de se utilizar a simulação em um processo logístico, a fim de encontrar possíveis gargalos operacionais e viabilizar uma otimização que proporcione um melhor uso dos recursos disponíveis, considerando as limitações de cada processo. Assim sendo, pode-se concluir dizendo que os objetivos para o estudo em questão foram atingidos, obtendo-se êxito na compreensão da dinâmica de funcionamento para o segmento de refrigerantes, assim como em oferecer um melhor planejamento para a empresa estudada neste presente trabalho, que busca a sua consolidação nesse ramo de negócios.

\section{REFERÊNCIAS}

AMORIN, L. G.Roteirização aplicada à indústria de bebidas. Monografia (relatório de estágio). Brasília DF, 2014. Disponível em:

<http://bdm.unb.br/bitstream/10483/9245/1/2014_LucasGomesAmorim.pdf>. Acesso em: 25 ago. 2018.

ARAÚJO, M. A. D. Administração de Produção e Operações, uma abordagem prática. $1^{\text {a }}$ ed. São Paulo: Armazém de Ideias, 2008.

BALLOU, R. H. Gerenciamento da Cadeia de Suprimentos / Logística Empresarial, $5^{\mathrm{a}}$ ed São Paulo: Bookman, 2006.

BARTON, R.F. A primer on simulation and gaming. Englewood Cliffs, New Jersey: Prentice-Hall, 1970.

DANTAS, E. M. A. Estágio da organização logística de três empresas do setor de bebidas:um estudo de caso. Dissertação de mestrado. Universidade federal do Rio de Janeiro, Rio de Janeiro: 2000. Disponível em: <

http://www.coppead.ufrj.br/upload/publicacoes/Eduardo_Dantas.pdf>. Acesso em: 3 set. 2018 
DONATO, J.V. Empreendedorismo e estratégia - estudo de criação de duas empresas no setor de refrigerantes no Ceará. Fortaleza: Banco do Nordeste, 2013.

Análise setorial: bebidas não alcoólicas. 2010. Disponível em:

http://d001wwv06/AmbEstudosPesqAval/AnalisesSetoriais/docs/901120810.doc Acesso em: 31 ago. 2018.

FERREIRA, F. M. Modelagem de sistemas mecânicos utilizando procedimentos modulares. Dissertação (Mestrado em Engenharia Mecânica) - Instituto Militar de Engenharia, Rio de Janeiro, 2006.

PARAGON. Introdução à Simulação. In: ENCONTRO NACIONAL DE ENGENHARIA DE PRODUÇÃO. Porto Alegre, 2005. Anais. Porto Alegre: ENEGEP, 2005.

PAULA, A.; HAIDUKE, I. F. O uso de novas tecnologias aplicadas ao processo de ensino e aprendizagem: O software ARENA. 2015. Disponível em:

$<$ https://slidex.tips/download/o-uso-novas-tecnologias-aplicadas-ao-processo-ensino-eaprendizagem-o-software-a>. Acesso em: 17 set. 2018.

FRANCISCHETTI, C. E. Aplicação de contabilometria no processo de planejamento econômico de uma empresa de varejo nacional. Disponível em:

$<$ https://www.unimep.br/phpg/bibdig/pdfs/docs/21032016_130101_carloseduardofrancischett i_ok.pdf >. Acesso em: 20 set. 2018.

MENTZER, J., DEWITT, W., KEEBLER, J., MIN, S., NIX, N., SIMTH, C. e ZACHARIA, Z. (2001).Defining Supply Chain Management, Journal of Business Logistics, Vol. 22, $\mathrm{n}^{\mathbf{0}} 2$, pp. $1-25$

SALIBY, E. Repensando a simulação: a amostragem descritiva. São Paulo: Atlas, 1989

SARAIVA JÚNIOR, A. F.; TABOSA, C. DE M.; COSTA, R. P. DA. Simulação de Monte Carlo aplicada à análise econômica de pedido. Produção, v. 21, n. 1, p. 149 - 164, 2011.

PRADO, D. Usando o ARENA em Simulação. 5. ed. Minas Gerais: Falconi, 2014. V.3. Usando o ARENA em simulação. 4.ed. Nova Lima: INDG LTDA, 2010.

VAN DER VORST, J. et al. QualityControlled Logistics in Food Supply Chain Network Integrated Decision-Making on Qualityand Logistics to Meet Advanced Customer Demands. In: Proceedings of the 14th InternationalEuroma Conference, 17-20 June, Ankara, Turkey, pp. 1-10. 2007. 